\title{
Kv1.3 voltage-gated potassium channels link cellular respiration to proliferation through a non- conducting mechanism
}

\author{
Faye L. Styles ${ }^{1}$, Moza M. Al-Owais², Jason L. Scragg ${ }^{1}$, Eulashini Chuntharpursat-Bon', Nishani T. Hettiarachchi', \\ Jonathan D. Lippiat', Aisling Minard ${ }^{3}$, Robin S. Bon $\mathbb{1}^{1}$, Karen Porter ${ }^{1}$, Piruthivi Sukumar ${ }^{1}$, Chris Peers ${ }^{1}$ and \\ Lee D. Roberts (i) ${ }^{1}$
}

\begin{abstract}
Cellular energy metabolism is fundamental for all biological functions. Cellular proliferation requires extensive metabolic reprogramming and has a high energy demand. The Kv1.3 voltage-gated potassium channel drives cellular proliferation. Kv1.3 channels localise to mitochondria. Using high-resolution respirometry, we show Kv1.3 channels increase oxidative phosphorylation, independently of redox balance, mitochondrial membrane potential or calcium signalling. Kv1.3-induced respiration increased reactive oxygen species production. Reducing reactive oxygen concentrations inhibited Kv1.3-induced proliferation. Selective Kv1.3 mutation identified that channel-induced respiration required an intact voltage sensor and C-terminal ERK1/2 phosphorylation site, but is channel pore independent. We show Kv1.3 channels regulate respiration through a non-conducting mechanism to generate reactive oxygen species which drive proliferation. This study identifies a Kv1.3-mediated mechanism underlying the metabolic regulation of proliferation, which may provide a therapeutic target for diseases characterised by dysfunctional proliferation and cell growth.
\end{abstract}

\section{Introduction}

Cellular energy metabolism is key to all biological processes. Biosynthesis of the macromolecular components of cells requires energy, generated in the mitochondria, in the form of ATP. Therefore redox regulation, and balancing the metabolic needs of oxidative phosphorylation (OXPHOS) required for ATP production and the generation of new lipids and nuclear material, is essential for cell proliferation. The mechanisms through which cells regulate their metabolism to drive cell growth and proliferation is not fully understood. These processes involve integrated environmental sensing and intracellular signalling, coupling the initiators of proliferation with the

\footnotetext{
Correspondence: Lee D. Roberts (L.D.Roberts@leeds.ac.uk)

${ }^{1}$ School of Medicine, University of Leeds, Leeds LS2 9JT, UK

${ }^{2}$ Faculty of Biological Sciences, University of Leeds, Leeds LS2 9JT, UK

Full list of author information is available at the end of the article

Edited by J.-E. Ricci
}

regulation of energy metabolism ${ }^{1,2}$. Here, we propose a role for a voltage-gated ion channel in the process linking proliferation and energy metabolism.

Voltage-gated potassium $(\mathrm{Kv})$ channels are transmembrane proteins that facilitate $\mathrm{K}^{+}$movement through membranes via their intrinsic pores. $\mathrm{K}^{+}$influx into a cell or organelle activates signalling cascades that can regulate proliferation $^{3}$, cell volume ${ }^{4}$, apoptosis, migration $^{5}$ and energetics $^{6,7}$. The Kv1.3 channel is present in a widerange of tissues including the brain ${ }^{8,9}$, epithelial cells ${ }^{10}$, adipose tissue ${ }^{6}$ and both skeletal and smooth muscle cells $^{11,12}$. Kv1.3 channels stimulate cellular proliferation $^{3,13-15}$ with mechanisms implicated including ion conducting effects on plasma membrane potential ${ }^{16}, \mathrm{Ca}^{2+}$ influx ${ }^{15}$ and cell volume regulation ${ }^{17}$. Non-conducting signalling mechanisms, independent of ion influx/efflux, are emerging. These arise due to interactions between the channels and signalling/scaffolding proteins that either

\section{(c) The Author(s) 2021}

(c) (i) Open Access This article is licensed under a Creative Commons Attribution 4.0 International License, which permits use, sharing, adaptation, distribution and reproduction cc) in any medium or format, as long as you give appropriate credit to the original author(s) and the source, provide a link to the Creative Commons license, and indicate if changes were made. The images or other third party material in this article are included in the article's Creative Commons license, unless indicated otherwise in a credit line to the material. If material is not included in the article's Creative Commons license and your intended use is not permitted by statutory regulation or exceeds the permitted use, you will need to obtain permission directly from the copyright holder. To view a copy of this license, visit http://creativecommons.org/licenses/by/4.0/. 
alter the channel's cellular location or couple the channel to intracellular second messengers ${ }^{18,19}$.

Cellular proliferation has a high energy demand, requiring ATP for processes ranging from signal transduction to DNA, RNA and protein synthesis ${ }^{20}$. Besides the plasma membrane, Kv1.3 has been reported at intracellular sites, including the nucleus ${ }^{21}$ and the $\mathrm{ER}^{22}$. Plasma membrane and intracellular Kv1.3 channels may have distinct roles in cellular homoeostasis ${ }^{18,23,24}$. Kv1.3 has also been found in the inner mitochondrial membrane, the site of ATP synthesis ${ }^{23,25,26}$.

We hypothesised that the Kv1.3 channel may coordinate the energy demands required for Kv1.3-stimulated proliferation. We identify that intracellular Kv1.3 channels function through a non-conducting mechanism to regulate cellular energetics and demonstrate that regulation of respiration by the channel is a stimulatory mechanism for Kv1.3-mediated proliferation.

\section{Materials and Methods}

Cell culture and generation of Kv1.3 and Kv1.5 cells

Wild type (WT) HEK293 (ECACC, Public Health England, Porton Down, UK) and HEK293/Kv1.3 cells were cultured in DMEM supplemented with $10 \%(\mathrm{v} / \mathrm{v})$ foetal bovine serum (BioSera, East Sussex, UK), 1\% (v/v) Antibiotic-Antimycotic $\quad(10,000$ units $/ \mathrm{mL}$ penicillin, $10,000 \mu \mathrm{g} / \mathrm{mL}$ streptomycin, $25 \mu \mathrm{g} / \mathrm{mL}$ Fungizone $\left.^{\mathrm{TM}}\right), 1 \%$ $(\mathrm{v} / \mathrm{v})$ non-essential amino acids, $0.1 \%(\mathrm{v} / \mathrm{v})$ gentamicin $(50 \mathrm{mg} / \mathrm{ml}), 1 \%(\mathrm{v} / \mathrm{v})$ GlutaMAX and geneticin (G418 Sulfate, $600 \mathrm{ug} / \mathrm{ml})\left(5 \% \mathrm{CO}_{2} ; 37^{\circ} \mathrm{C}\right)$. All cell culture reagents were purchased from Gibco-BRL (Paisley, UK) unless otherwise stated.

HEK293/Kv1.3 cells, and plasmids containing cDNA encoding mutant Kv1.3 channels were a gift from Professors José Ramón López López and Teresa Pérez-García (University of Valladolid). Kv1.3-P89 is a voltage sensitive, non-conducting channel containing a point mutation (W389F) in the S5-S6 linker of the Kv1.3-GFP fusion sequence (Supplementary Fig. S1A). Kv1.3-P93 is a nonconducting, voltage-insensitive channel and was generated as for Kv1.3-P89 but with three additional mutations (R320N/L321A/R326I) in the S4 (voltage sensor) region (Supplementary Fig. S1B). Kv1.3-P118 is a Kv1.3-mCherry fusion protein. Kv1.3-P121 is a phosphorylation-defective Kv1.3-mCherry fusion protein containing a point mutation (Y447A) in Kv1.3's C-terminal region (Supplementary Fig. S1C $)^{13,18}$. Plasmid maps are shown in Supplementary Fig. S2 (Supplementary Tables 1 and 2).

WT HEK293 cells were transfected with plasmid DNA (control HEK293) or plasmid DNA containing Kv1.3, or mutant Kv1.3 channels by nucleofection using Cell Line Nucleofector Kit V and Nucleofector ${ }^{\mathrm{TM}}$ Programme Q-01 (Lonza, Castleford, UK). Transfected cells were selected with G-418 antibiotic $(1 \mathrm{mg} / \mathrm{ml}), 2$ days after transfection.
Selection was applied for 4 weeks, colonies picked, grown to confluence and screened electrophysiologically. Stable expression of mutant Kv1.3 channels was confirmed using immunoblotting (Supplementary Fig. S3).

Kv1.5 cDNA was amplified from a human foetal brain cDNA library (Clontech, UK) and HEK293/Kv1.5 cells were engineered to express the Kv1.5 channel (KCNA5) as described ${ }^{27}$.

\section{Proliferation assays}

Proliferation assays followed established protocols ${ }^{28-30}$. Cells were seeded at $2 \times 10^{4}$ cells per well of a 24-well plate. After $6 \mathrm{~h}$ media was replaced with serum-free media (SFM) overnight. On day 0 , SFM was removed and growth media $(1 \mathrm{ml})$ was added to each well. For pharmacological studies, ShK-Dap22 (1-200 pM), PAPTP (100 nM), PAP$1(100 \mathrm{nM})$ or MitoQ $(5 \mu \mathrm{M})$ were added on day 0 . Cells were counted in triplicate on days $0,1,2$ and 3 using a Bio-Rad TC10 cell counter (Bio-Rad Laboratories, USA).

\section{High-resolution respirometry}

Respirometry (Oxygraph-2K; Oroboros Instruments, Austria $^{31}$ was performed in intact HEK293 cells using established methods ${ }^{32}$. Briefly, $1 \times 10^{6}$ cells $/ \mathrm{cm}^{3}$ in SFM were added to the respiratory chambers and routine respiration measured. Non-phosphorylating leak was determined ( $2 \mu \mathrm{g} / \mathrm{ml}$ oligomycin). Maximum uncoupled electron transfer system (ETS) was measured using carbonyl cyanide$p$-trifluoromethoxyphenylhydrazone (FCCP; $0.5 \mu \mathrm{M})$. Rotenone $(2.5 \mu \mathrm{M})$ and Antimycin $\mathrm{A}(0.5 \mu \mathrm{M})$ were added to determine residual oxygen consumption (ROX). Complex IV activity was assessed using ascorbate $(0.5 \mathrm{mM})$ and $N, N$, $N, \quad N^{p}$-Tetramethyl-p-phenylenediamine dihydrochloride ( $2 \mathrm{mM})$. Sodium azide $(20 \mathrm{mM})$ was added to inhibit mitochondrial respiration ${ }^{33}$.

\section{Citrate synthase assay}

Citrate synthase assay followed described methods ${ }^{34}$.

\section{Immunocytochemistry}

Cells were cultured on poly-L-lysine coated coverslips until 30-40\% confluent. Control HEK293 and HEK293/ Kv1.3 cells were fixed ( $4 \%$ paraformaldehyde, $20 \mathrm{~min}$ ), washed $(3 \times 1 \mathrm{ml}$ DPBS $)$ and incubated in DPBS $(0.05 \%$ Triton X100, 10\% NGS; $20 \mathrm{~min})$. Cells were washed (DPBS, 1\% NGS) Anti-Kv1.3 Clone L23/27, mouse monoclonal (1:500; cat no. 75-009, NeuroMab, Antibodies Incorporated, UK) or Anti-ATPB rabbit polyclonal (1:500; cat no. ab128743, Abcam, UK) antibody were added in 1\% NGS and incubated in a humidified chamber overnight at $4{ }^{\circ} \mathrm{C}$. Coverslips were washed with DPBS with $1 \%$ NGS $(3 \times 1 \mathrm{ml})$. Alexa Fluor 488 Donkey anti-Mouse IgG (cat no. R37114, ThermoFisher) secondary antibody or HRP goat anti-rabbit IgG $\mathrm{H}+\mathrm{L}$ secondary antibody (cat no. 
926-80011 LI-COR Biosciences UK Ltd, Cambridge, UK) was added (1:2000 in 1\% NGS) for $1 \mathrm{~h}$. Cells were washed with DPBS with $1 \%$ NGS $(3 \times 1 \mathrm{ml})$, and mounted with VECTASHIELD Mounting Medium with DAPI (VECTOR Laboratories Ltd, UK). Mitochondria were imaged following incubation with MitoTracker Red CMXRos (ThermoFisher) $\left(100 \mathrm{nM} ; 30 \mathrm{~min} ; 37^{\circ} \mathrm{C} ; 5 \% \mathrm{CO}_{2}\right)$. Excitation/emission wavelengths: $579 / 599 \mathrm{~nm}$. Imaging was perfomed using a Zeiss LSM800 microscope and processed using Zen Lite 64 (Zeiss, UK) and Image J.

\section{Cellular ROS assays}

Cells were treated with $5 \mu \mathrm{M}$ CellROX Deep Red (ThermoFisher Scientific, UK) $\left(30 \mathrm{~min} ; 37^{\circ} \mathrm{C} ; 5 \% \mathrm{CO}_{2}\right.$ ) with or without a $100 \mu \mathrm{M}$ menadione $1 \mathrm{~h}$ pretreatment.

\section{Electrophysiology}

Cells were perfused at $2-4 \mathrm{~mL} / \mathrm{min}$ (volume $80 \mu \mathrm{L}$ ) with recording solution $(\mathrm{NaCl} 141 \mathrm{mM}$, HEPES $10 \mathrm{mM}, \mathrm{KCl}$ $4.7 \mathrm{mM}, \quad \mathrm{MgCl}_{2} 1.2 \mathrm{mM}, \mathrm{CaCl}_{2} 1.8 \mathrm{mM}, \quad \mathrm{D}$-glucose $10 \mathrm{mM}, \mathrm{pH} 7.4,21-24^{\circ} \mathrm{C}$ ) in a recording chamber mounted on an Olympus CK40 inverted microscope. Whole-cell patch-clamp recordings were made using patch pipettes (3-7 M $\Omega$ resistance) with electrode solution (EGTA $10 \mathrm{mM}$, HEPES $10 \mathrm{mM}, \mathrm{KCl} 125 \mathrm{mM}, \mathrm{MgCl}_{2}$ $4 \mathrm{mM}$ and MgATP $5 \mathrm{mM}, \mathrm{pH}$ 7.2). Experiments were recorded on an Axopatch 200B amplifier (ALA Scientific Instruments, NY, USA), and stored and digitised using a Digidata 1322A (Axon, Molecular Devices, USA) and pClamp 10 (Molecular Devices), respectively. Two protocols were adopted: (1) Current-voltage (I/V) relationships measured by stepping from $-80 \mathrm{mV}$ holding potential to voltages between -60 and $+60 \mathrm{mV}$ in $10 \mathrm{mV}$ increments for $300 \mathrm{~ms}$ and (2) a single step from -80 to $+40 \mathrm{mV}$ for $200 \mathrm{~ms}$.

\section{Cairn photometry}

Cells were cultured on poly-L-lysine coated coverslips until $90 \%$ confluent, washed (DPBS, $2 \times 2 \mathrm{ml}$ ), treated with $20 \mathrm{nM}$ tetramethylrhodamine, methyl ester (TMRM) (30 min, $5 \% \mathrm{CO}_{2}, 37^{\circ} \mathrm{C}$ ), washed (DPBS, $2 \times 2 \mathrm{ml}$ ) and placed in Hank's balanced salt solution. Coverslips were transferred to the recording chamber of an inverted epifluorescence microscope and perfused with recording solution (as for electrophysiology). Recordings were made using a Cairn Research ME-SE Photometry system (Cairn Research, UK). TMRM was excited by a Xeon Arc lamp (excitation/emission wavelengths: 548/574 nm).

\section{Flow cytometry}

$\mathrm{Ca}^{2+}$ levels were measured using RHOD-2AM (20 nM) at $548 / 574 \mathrm{~nm}$ (excitation/emission wavelengths); mitochondrial membrane potential (MMP) was investigated using TMRM $(1 \mu \mathrm{M})$ at $552 / 581 \mathrm{~nm}$ and NADH autofluorescence was measured at $350 / 470 \mathrm{~nm}$. Gates were applied using unstained controls. Cells were analysed on a BD LSRFortessa ${ }^{\mathrm{TM}}$ analyser (BD Biosciences, USA) using the BD FACSDiva software (BD Biosciences, USA).

\section{Immunoblotting}

Cells were lysed with $\mathrm{M}$-per ${ }^{\mathrm{TM}}$ (Perbio Science, UK) containing mini protease inhibitors (Roche Diagnostics UK, UK). Protein concentrations were determined using a BCA assay (Pierce, USA). Cell lysate was added to sample buffer (1:5; $60 \mathrm{mM}$ Tris-Cl pH 6.8, 2\% SDS, 10\% glycerol, $5 \% \beta$-mercaptoethanol, $0.01 \%$ bromophenol blue). Samples $(10-20 \mu \mathrm{g}$ protein) were loaded on to $12.5 \%$ polyacrylamide-SDS gels (Bio-Rad, UK) and separated at $200 \mathrm{~V}$ (45 min), then transferred to polyvinyl difluoride membranes $\left(30 \mathrm{~V}\right.$, overnight at $4{ }^{\circ} \mathrm{C}$; Millipore Corporation, Massachusetts, USA). Membranes were blocked with $5 \%$ non-fat milk protein in TBS-Tween (TBST, $0.05 \% ; 1 \mathrm{~h}$ ) and treated with antibodies: Anti-Kv1.3 clone L23/27 mouse monoclonal primary antibody (1:500; cat no. 75009, NeuroMab, USA); Anti-COX IV mouse monoclonal primary antibody (1:500; cat no. 33985 Abcam, UK); Anti $\beta$-Actin Clone AC-15 mouse monoclonal primary antibody (1:600; cat no. A1978, Sigma Aldrich, UK); AntiATPB rabbit polyclonal antibody (1:500; cat no. 65378 Abcam, UK) diluted in 1\% non-fat milk protein in TBST $(3 \mathrm{~h})$. Membranes were washed $(6 \times 5 \mathrm{~min}$ in TBST $)$ and incubated $(1 \mathrm{~h})$ with HRP-conjugated goat anti-mouse IgG $\mathrm{H}+\mathrm{L}$ secondary antibody for Anti-Kv1.3, Anti-COX IV and Anti $\beta$-Actin (1:20000; cat no. 926-80010, LI-COR Biosciences, UK) or HRP-conjugated goat anti-rabbit IgG $\mathrm{H}+\mathrm{L}$ secondary antibody for Anti ATBP (1:20000; cat no. 926-80011, LI-COR Biosciences, UK). Membranes were washed in TBST $(3 \times 5 \mathrm{~min})$, before annotation with a LI-COR WesternSure pen (LI-COR Biosciences, USA). A 1:1 mixture of LI-COR Western Sure ECL luminol enhancer solution with LI-COR Western Sure ECL stable peroxide was used to wash the membranes $(5 \mathrm{~min})$. Membranes were imaged on a C-Digit Blot Scanner (LICOR Biosciences, UK) (12 min). Densitometry was quantified in Image J.

\section{Chemicals}

PAPTP was synthesised according to literature ${ }^{26}$ and purified (assessed by 1H-NMR and 13C-NMR, Supplementary Fig. S4A, B).

\section{Statistical analysis}

Sample sizes were calculated using power calculations. Where sample sizes were not calculated sample size was considered adequate based on the size and reproducibility of between group differences. Samples were randomly assigned to experimental groups. Group variance was 
analysed with an $F$-test. Data were analysed using either a paired, or unpaired two-tailed Student's $t$-test and/or oneway and two-way ANOVA with a Sidak's or Tukey's post hoc test. Significance was determined when $p \leq 0.05$.

\section{Results}

Kv1.3 expression increases cellular proliferation and respiration

HEK293 cells were transfected to express the Kv1.3 channel. Immunocytochemistry immunoblotting and electrophysiology (Supplementary Fig. S5A-F) confirmed that the HEK293/Kv1.3 cells expressed functional Kv1.3. HEK293/Kv1.3 cells exhibited increased proliferation compared to control HEK293 cells (Fig. 1A).

High-resolution respirometry identified that Kv1.3 expression increased mitochondrial respiration in HEK293 cells (Fig. 1B). Kv1.3 expression increased Routine, non-phosphorylating proton Leak, maximal uncoupled ETS and non-OXPHOS ROX respiration. When corrected for ROX, Routine and maximal respiration remained elevated in Kv1.3-expressing cells (Fig. 1C). The ATP turnover (Fig. 1D) and spare respiratory capacity (Fig. 1E) were increased by Kv1.3 expression.

In contrast to Kv1.3, expression of the voltage-gated $\mathrm{K}^{+}$ channel Kv1.5 reduces proliferation ${ }^{35}$. Respirometry was used to determine whether Kv1.5 also has contrasting effects on respiration in HEK293 cells expressing Kv1.5. HEK293/Kv1.5 cells exhibited reduced proliferation (Fig. 1F) and had lower Routine/basal and Leak respiration compared to control HEK293 or HEK293Kv1.3 cells, independent of ROX (Fig. 1G, H). The opposing effects on proliferation by Kv1.3 and Kv1.5 channel expression are mirrored by effects on mitochondrial respiration.

\section{Plasma membrane Kv1.3 channel $\mathrm{K}^{+}$transport does not regulate proliferation or respiration}

Next we determined whether $\mathrm{K}^{+}$transport by $\mathrm{Kv} 1.3$ channels in the plasma membrane regulated the increase in cellular proliferation and respiration. ShK-Dap22 is a potent, membrane impermeable, Kv1.3 inhibitor ${ }^{36}$. Electrophysiology recordings confirmed that ShK-Dap22 inhibited plasma membrane Kv1.3 in HEK293/Kv1.3 cells (Fig. 2A). The $\mathrm{IC}_{50}$ of ShK-Dap22 for Kv1.3 current inhibition is $39.6 \mathrm{pM}^{36}$. ShK-Dap22 $(80 \mathrm{pM})$ reduced the potassium currents in HEK293/Kv1.3 cells. A concentration-response was used to assess the effect of ShK-Dap22 (1-200 pM) on proliferation of control HEK293 (Fig. 2B) and HEK293/ Kv1.3 cells (Fig. 2C). ShK-Dap22 did not affect proliferation. $\mathrm{K}^{+}$transport through plasma membrane $\mathrm{Kv1} .3$ does not induce channel-dependent proliferation.

We then assessed whether plasma membrane Kv1.3 channels induce mitochondrial respiration. HEK293/ Kv1.3 cells were treated with ShK-Dap22 and analysed with respirometry. ShK-Dap22 up to 500 pM had no effect on Routine, Leak, ETS or ROX respiration in HEK293/ Kv1.3 cells (Fig. 2D). Plasma membrane Kv1.3 channel conductance does not induce channel-dependent mitochondrial respiration.

\section{Kv1.3 channels localise to the mitochondria but do not increase cellular mitochondrial content}

The Kv1.3-induced increase in mitochondrial respiration was not activated by $\mathrm{K}+$ transport through plasma membrane Kv1.3 channels. Kv1.3 has been reported to localise to the mitochondrial membrane ${ }^{23,25,26}$. We investigated whether Kv1.3 channels localise to the mitochondria in our model. Immunocytochemistry was employed to investigate Kv1.3 cellular localisation in control HEK293 and HEK293/Kv1.3 cells. Cells were stained for Kv1.3 (anti-Kv1.3 antibody and fluorescent secondary reporter; green), and mitochondria (MitoTracker CMXRos; red) and imaged using confocal microscopy (Fig. 3A). HEK293 cells do not exhibit autofluorescence within the MitoTracker detection wavelengths in cells not stained with MitoTracker (Supplementary Fig. S6A, B). As a secondary approach, HEK293 cells expressing mCherry-tagged Kv1.3 channels (HEK293/Kv1.3-P118; green) were immunofluorescently stained for mitochondrial complex IV (red) (Fig. 3A). In both cases Kv1.3 and mitochondria colocalisation was visualised in yellow and observed using a mitochondrial and Kv1.3 florescence intensity plotted line profile through a cross section of the images. HEK293/ Kv1.3 cells had greater Kv1.3 staining. The Kv1.3 channels were observed to co-localise with mitochondria. Interestingly, Kv1.3 expression is not ubiquitously distributed among mitochondria. Consistent with findings in the literature, our data suggest that the majority of the Kv1.3 channel protein is intracellular ${ }^{13}$.

Next we investigated whether increased respiration in Kv1.3-expressing cells was driven by increased mitochondrial content. Canonical biochemical markers of mitochondrial content, complex IV activity using respirometry (Fig. 3B) and citrate synthase activity (Fig. 3C) were determined in control HEK293 and HEK293/Kv1.3 cells. Expression of the mitochondrial protein ATP synthase was also examined using immunoblotting in HEK293/Kv.13 cells (Fig. 3D and Supplementary Fig. S6C). Kv1.3 expression did not increase cellular mitochondrial content.

We reasoned that Kv1.3-induced increases in mitochondrial respiration may occur via alterations to the mitochondrial network. Confocal microscopy was used to investigate the mitochondrial network of HEK293/ Kv1.3 cells. Cells were either stained with Mitotracker (red) (Fig. 3E) or immunostained for mitochondrial complex IV (red) (Fig. 3F). The gross structure of the mitochondrial network was unchanged by Kv1.3 expression. Kv1.3 does not exert its effects on cellular 

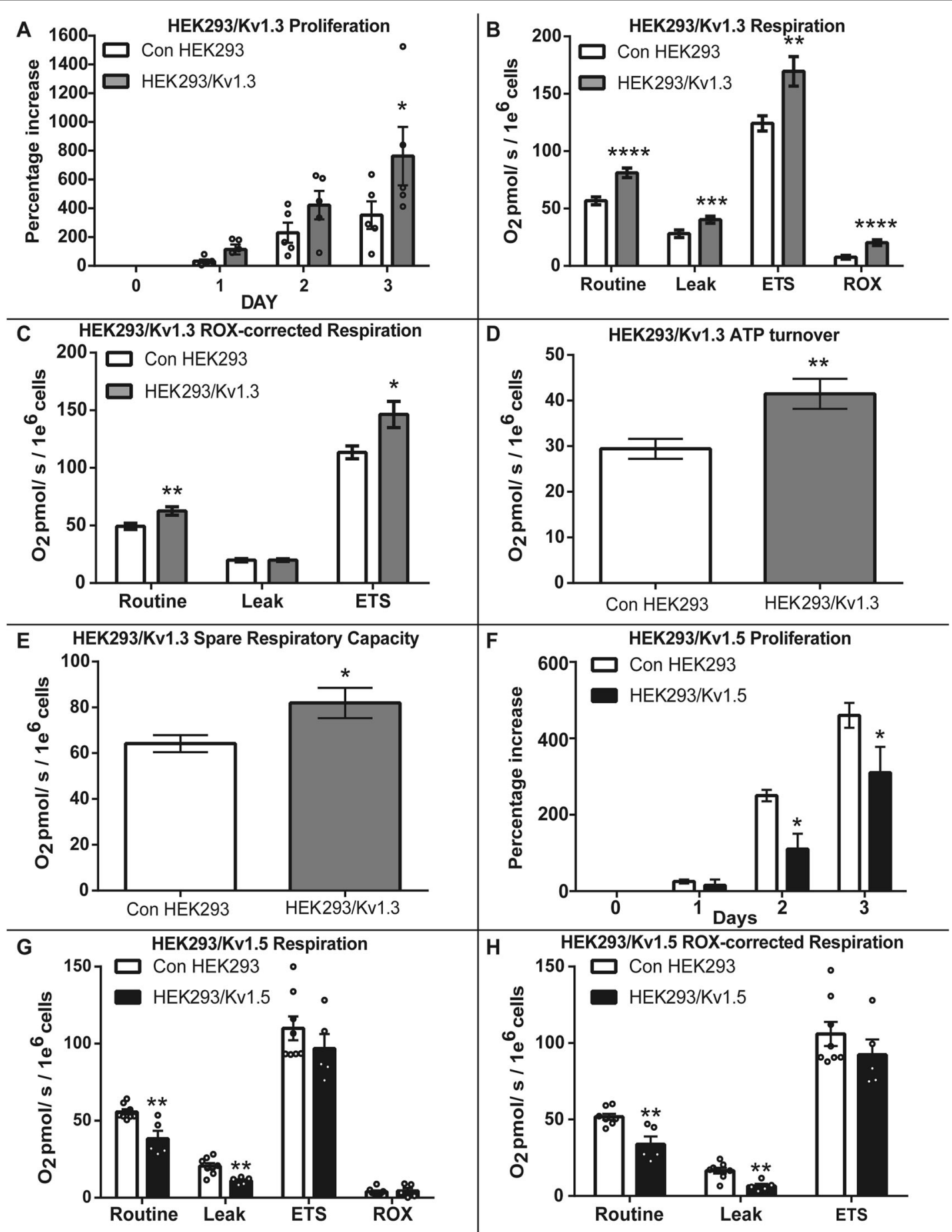

Fig. 1 Kv1.3 expression increases cellular proliferation and respiration. A HEK293/Kv1.3 cells exhibit enhanced proliferation compared with control HEK293 cells. The percentage increase in cell number over 3 days normalised to day $0(n=5)$. B Routine, Leak, maximal electron transfer system (ETS) and residual (ROX) $\mathrm{O}_{2}$ consumption in HEK293/Kv1.3 $(n=38)$ and control HEK293 ( $\left.n=27\right)$ cells measured by high-resolution respirometry. C ROX-corrected Routine, Leak and ETS O 2 consumption in HEK293/Kv1.3 ( $n=38$ ) and control HEK293 ( $n=27$ ) cells measured by highresolution respirometry. D ATP turnover in HEK293/Kv1.3 $(n=38)$ cells and control HEK293 $(n=27)$ cells. E Spare respiratory capacity in HEK293/Kv1.3 $(n=38)$ and control HEK293 $(n=27)$ cells. F Proliferation is suppressed in HEK293/Kv1.5 cells $(n=12)$ compared with control HEK293 $(n=15)$. Percentage increase in cell number at day 1, 2 and 3 from day 0. G Routine, Leak, ETS and ROX O 2 consumption in HEK293/Kv1.5 ( $n=5$ ) and control HEK293 ( $n=8$ ) cells measured by high-resolution respirometry. $\mathbf{H}$ ROX-corrected Routine, Leak, and ETS O 2 consumption in HEK293/Kv1.5 ( $n=5$ ) and control HEK293 $(n=8)$ cells measured by high-resolution respirometry. Data expressed as the mean \pm SEM. Data were analysed using either Student's t-test or two-way ANOVA. ${ }^{*} p<0.05,{ }^{* *} p<0.01,{ }^{* * *} p<0.001$ and ${ }^{* * *} p<0.0001$. 


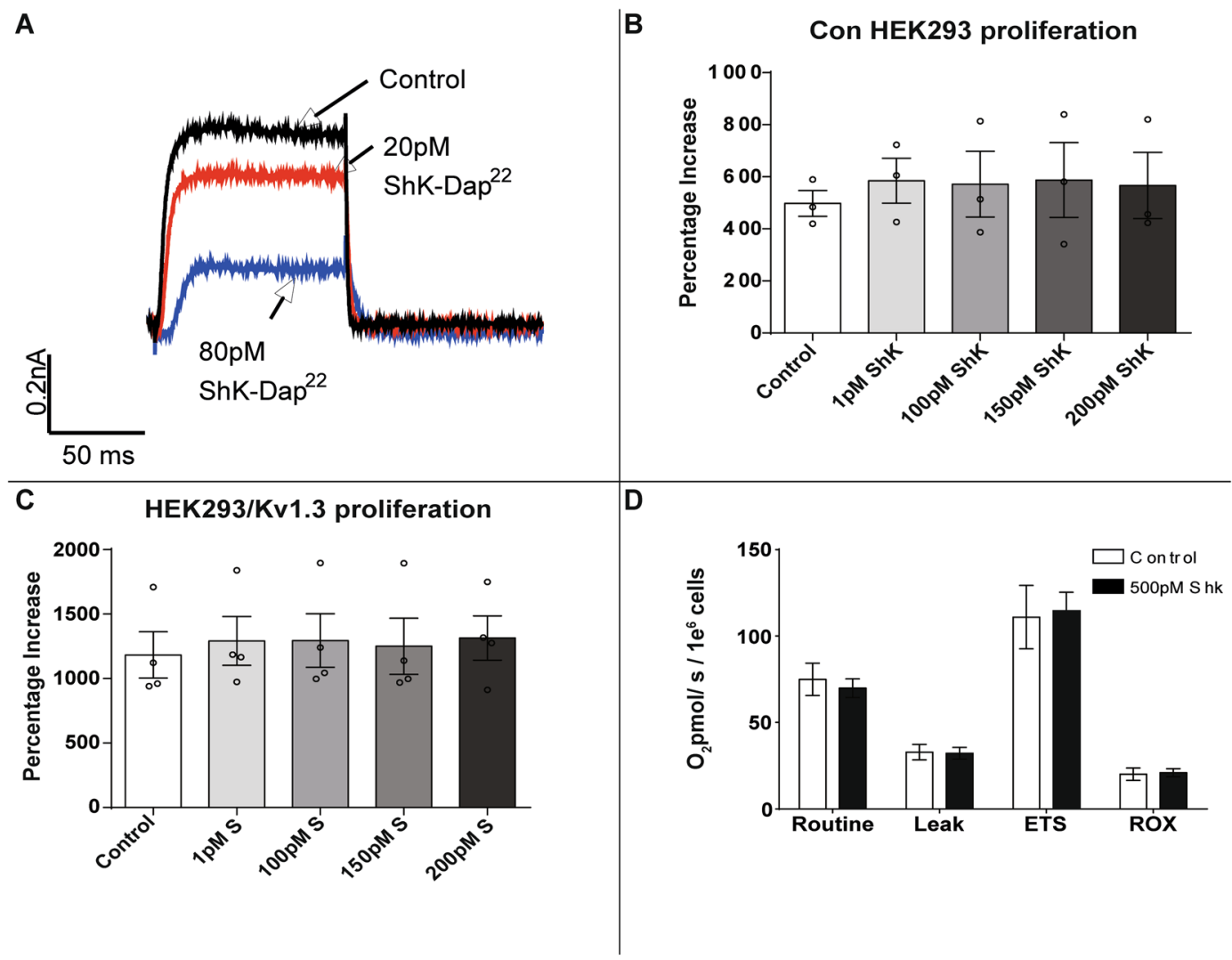

Fig. 2 Plasma membrane Kv1.3 channels do not regulate proliferation or respiration. A Whole cell voltage clamp trace showing inhibition of Kv1.3 currents by ShK-Dap22 (20 and 80 pM). B Proliferation of control HEK293 cells treated with ShK-Dap22 (1-200 pM). The percentage increase in cell number over 3 days normalised to day $0(n=3)$. C Proliferation of HEK293/Kv1.3 cells treated with ShK-Dap22 (1-200 pM). The percentage increase in cell number over 3 days normalised to day $0(n=4)$. D Routine, Leak, maximal electron transfer system (ETS) and residual $(R O X) \mathrm{O}_{2}$ consumption in HEK293/Kv1.3 cells treated with 500 pM ShK-Dap22 $(n=10)$. Data expressed as mean \pm SEM.

respiration through mitochondrial biogenesis or network reorganisation.

\section{Intracellular Kv1.3 channels regulate proliferation and respiration}

Plasma membrane Kv1.3 ion conductance did not regulate Kv1.3-induced respiration. We investigated the role of intracellular Kv1.3 channels in the regulation of proliferation and respiration. PAP-1 is a membrane permeable inhibitor of intracellular Kv1.3 channels, including those located at the cis-Golgi, ER, nucleus and mitochondria $^{21,22}$. PAP-1 functions by stabilising Kv1.3's inactivate state ${ }^{21,22}$. PAP-1 can be bound to triphenyl phosphonium lipophilic cation, forming the Kv1.3 inhibitor PAPTP, which inhibits the Kv1.3 whole-cell current and is pharmacologically targeted to the mitochondria $^{26,37,38}$. PAPTP was used to probe the role of the intracellular ion channels in proliferation and mitochondrial respiration. PAPTP $(100 \mathrm{nM})$ significantly reduced HEK293/Kv1.3 cell proliferation (Fig. 4A). The observed decrease in proliferation was not due to PAPTP cytotoxicity (Supplementary Fig. S7). We compared the effect of the mitochondrially-targeted Kv1.3 inhibitor PAPTP (100 nM) on HEK293/Kv1.3 cell proliferation to its nonspecific intracellular equivalent PAP-1 (100 nM) (Supplementary Fig. S8). The mitochondrially-targeted compound PAPTP had a greater inhibitory effect on HEK293/ Kv1.3 proliferation.

The effect of mitochondrially-targeted Kv1.3 channel inhibition on respiration rate was examined in HEK293/ Kv1.3 cells using PAPTP (Fig. 4B). PAPTP reduced Routine and maximal ETS respiration, whilst increasing proton Leak respiration. PAPTP also reduced both ATP turnover rate and spare respiratory capacity in HEK293/ Kv1.3 cells (Fig. 4C, D). PAPTP did not affect proliferation, or Routine, maximal ETS and Leak respiration in control HEK293 cells (Supplementary Fig. S9), indicating that PAPTP's effects in HEK293/Kv1.3 cells are not due to off-target activity. These data suggest that mitochondrial Kv1.3 channels may regulate proliferation and respiration. 


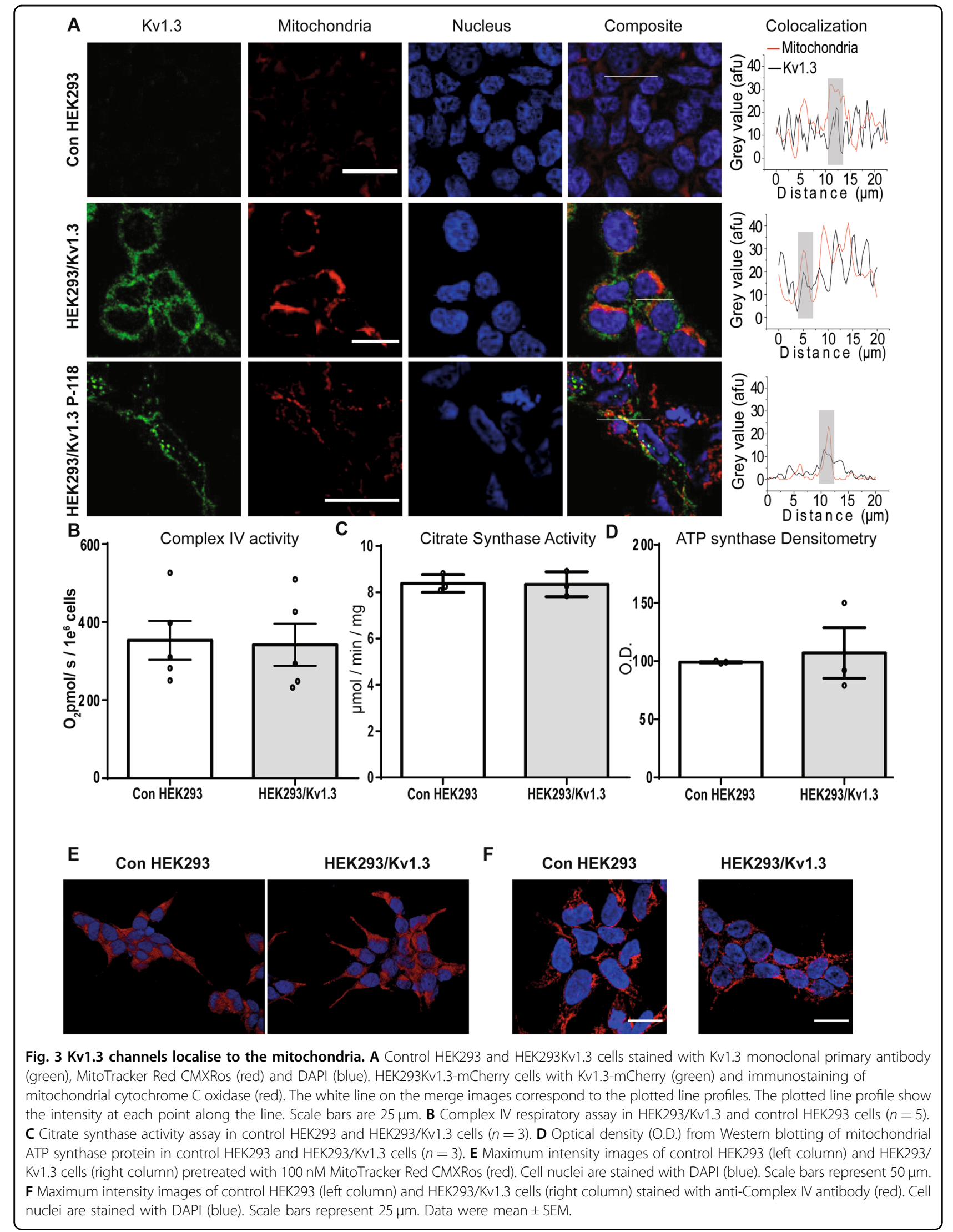




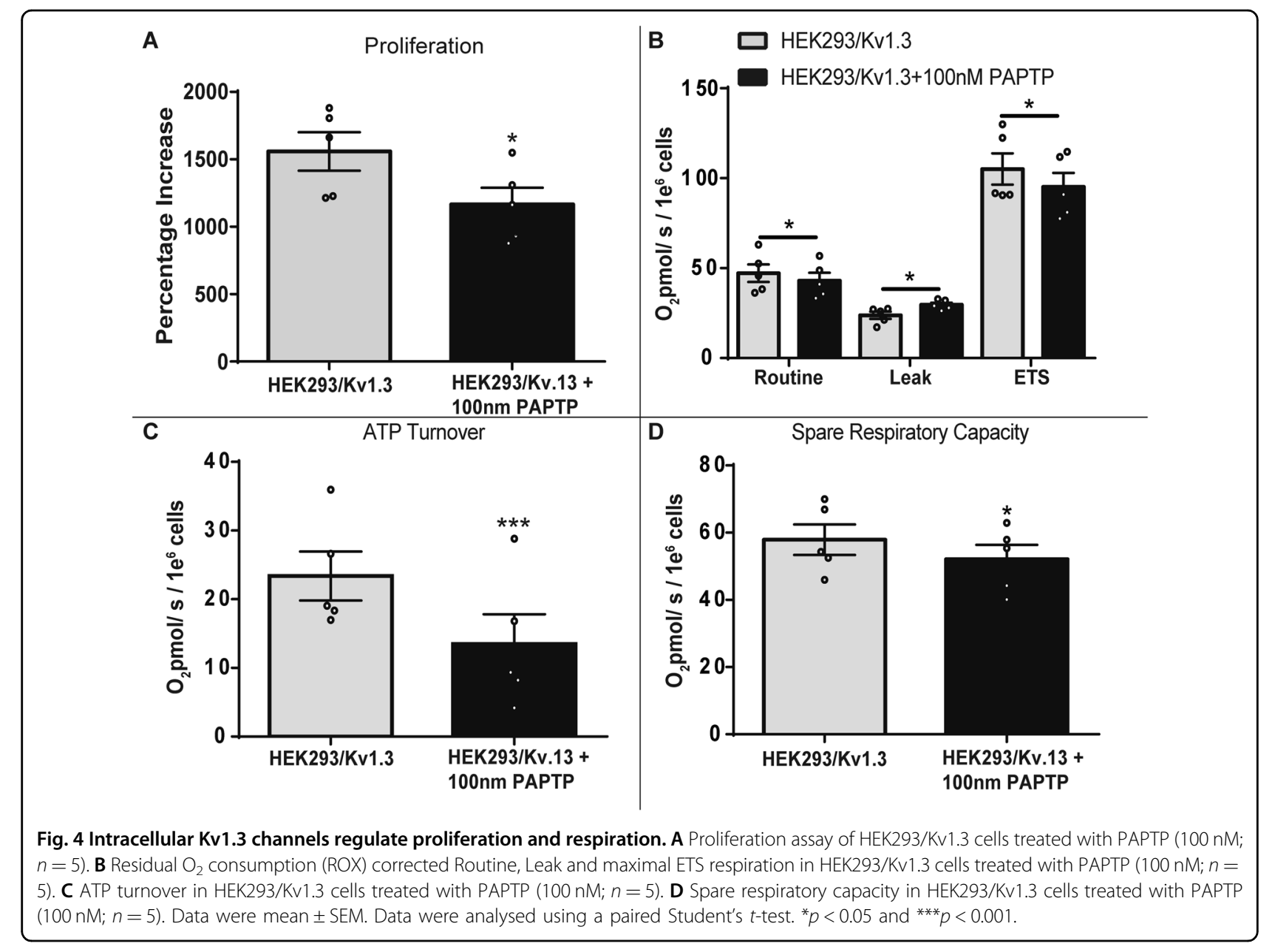

Reactive oxygen species generation links Kv1.3-induced respiration to cellular proliferation

Mitochondria are the primary site of cellular reactive oxygen species (ROS) production. Kv1.3 expression increases mitochondrial respiration. HEK293/Kv1.3 cells displayed greater ROS concentrations than control HEK293 cells, determined using the cell permeable fluorescent ROS dye CellROX Deep Red (Fig. 5A, B). Menadione induces cellular ROS generation through redox cycling. ROS production in response to $100 \mu \mathrm{M}$ menadione was greater in HEK293/Kv1.3 cells than in control HEK293 cells (Fig. 5C). These data suggest that increased mitochondrial respiration in HEK293/Kv1.3 cells leads to a greater capacity for ROS production. ROS can enhance cellular proliferation ${ }^{39}$. We investigated whether mitochondrial ROS in HEK293/Kv1.3 cells was driving the proliferative phenotype. MitoQ, a mitochondrially-targeted ROS scavenger, had no effect on cell viability (Fig. 5D), and significantly reduced proliferation in HEK293/Kv1.3 cells without affecting proliferation in control HEK293 cells (Fig. 5E). The increased respiration in Kv1.3 expressing cells increases capacity for
ROS generation, which in turn drives the proliferative phenotype.

\section{$\mathrm{Kv} 1.3$ regulates respiration independently of} mitochondrial membrane potential, $\mathrm{Ca}^{2+}$ or $\mathrm{NADH}$ redox status

We explored the mechanisms through which Kv1.3 regulates respiration. Mitochondrial ion channels can regulate the MMP to control respiration ${ }^{40}$. The MMP of HEK293/Kv1.3 and control HEK293 cells was investigated using the Cairn Photometry system. TMRM is a cellpermeant dye attracted to the negative charge of the mitochondrial membrane. TMRM accumulation is greater in HEK293/Kv1.3 cells, indicating greater MMP hyperpolarisation (Supplementary Fig. S10A). HEK293/ Kv1.3 and control HEK293 cells were sorted by flow cytometry following either treatment with TMRM, or TMRM and the OXPHOS uncoupler FCCP. Flow cytometry confirmed Kv1.3-induced increases in HEK293 cells MMP (Supplementary Fig. S10B). FCCP dissipates the MMP releasing mitochondrial TMRM. There was a greater difference in the mean fluorescent intensity 
A

\section{ROS Con HEK293}

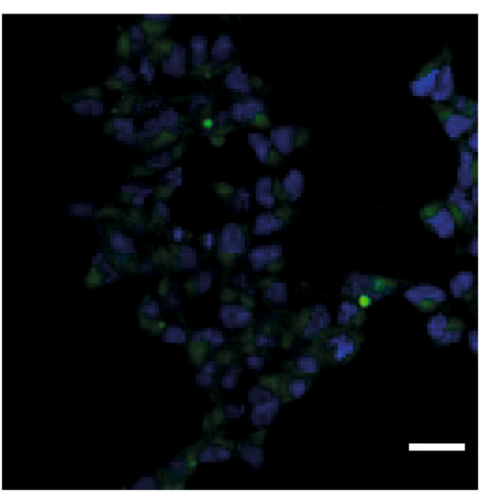

ROS HEK293/Kv1.3

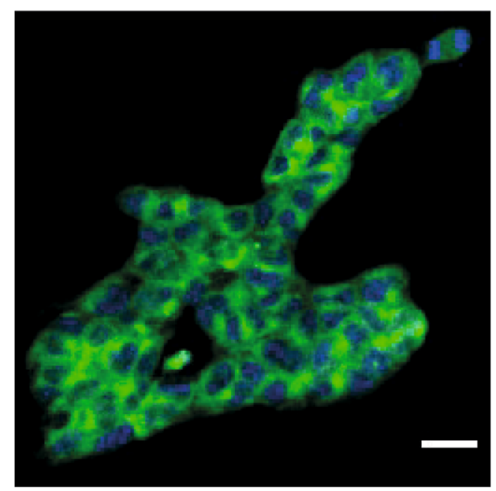

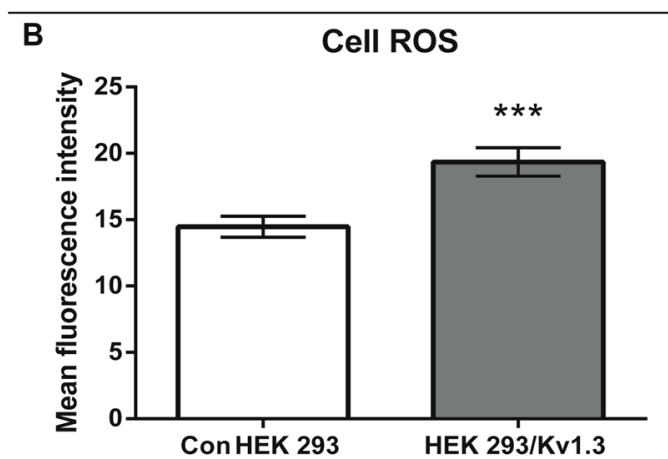
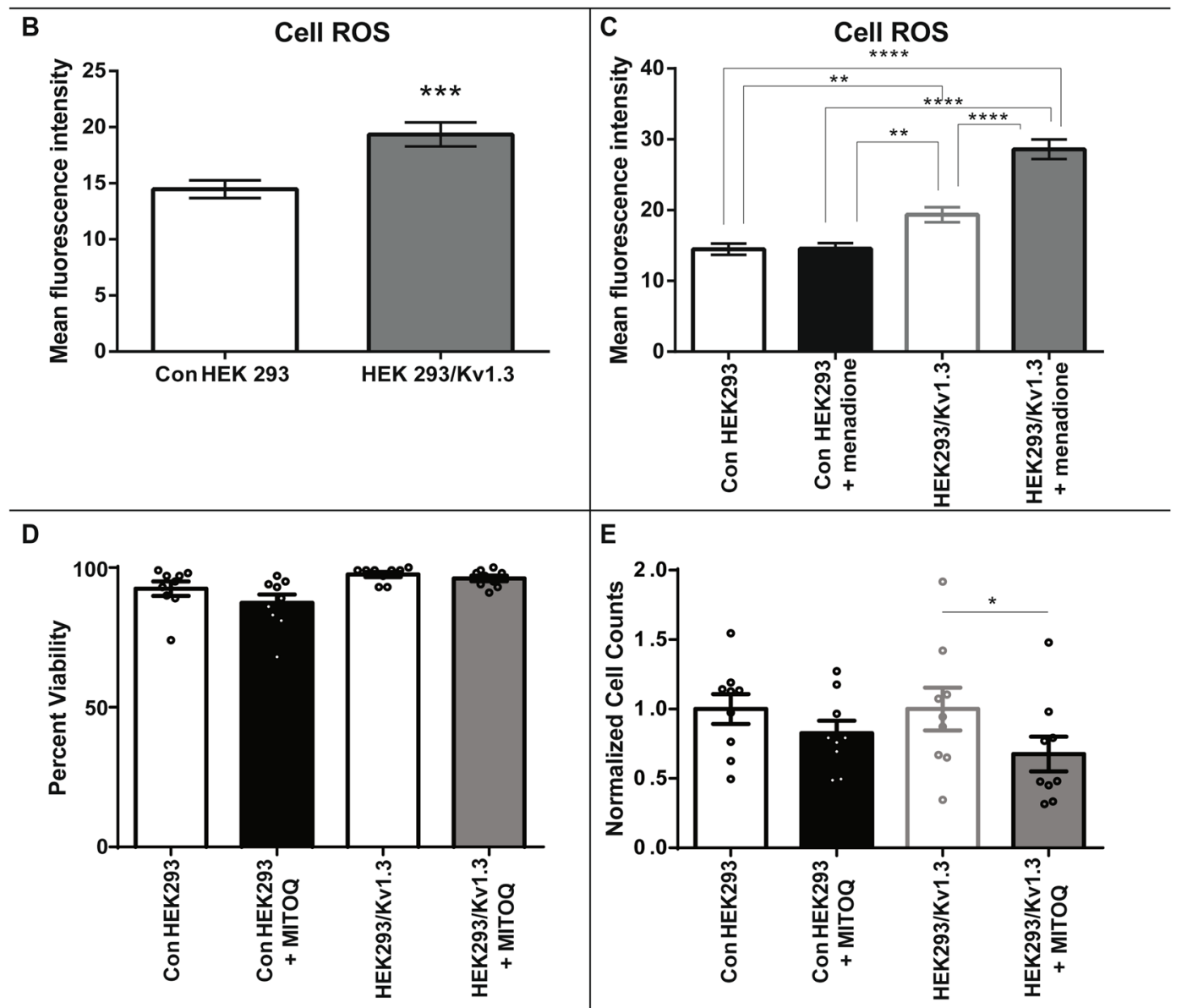

E

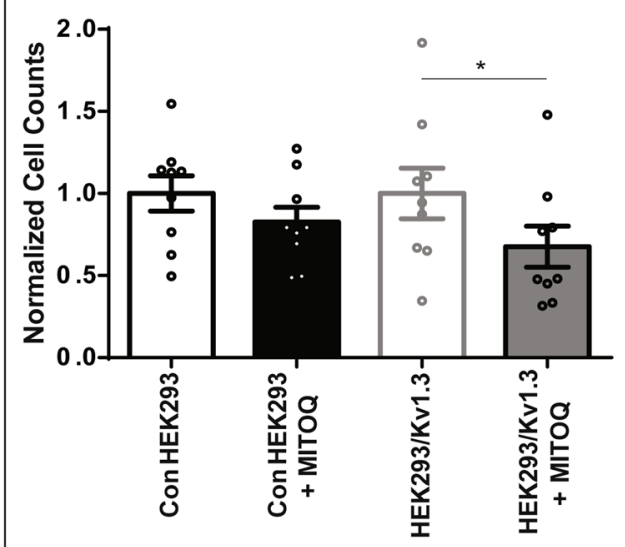

Fig. 5 Reactive oxygen species link mitochondrial Kv1.3-induced respiration to cellular proliferation. A Representative images showing cellular reactive oxygen species (ROS) in control HEK293 (left) and HEK293/Kv1.3 (right) cells stained with 5 HM CellROX deep red reagent (green) and DAPI to highlight cell nuclei (blue). Scale bars represent $25 \mu \mathrm{m}$. B ROS levels in control HEK293 cells and HEK293/Kv1.3 cells stained with $5 \mu$ M CellROX deep red reagent $(n=30)$. C ROS levels in control HEK293 and HEK293/Kv1.3 cells stained with $5 \mu$ M CellROX deep red reagent with and without $100 \mu \mathrm{M}$ menadione pretreatment $(n=30)$. D Percentage viability of control HEK293 and HEK293/Kv1.3 cells with and without $5 \mu \mathrm{M}$ MitoQ treatment assessed using trypan blue staining $(n=9)$. E Control HEK293 and HEK293/Kv1.3 cell counts following 3 days of proliferation, normalised to control, with and without $5 \mu \mathrm{M}$ MitoQ $(n=9)$. Data were expressed as mean \pm SEM and was analysed using either a Student's $t$-test or two-Way ANOVA with Tukey's post hoc test, ${ }^{*} p<0.05,{ }^{* *} p<0.01,{ }^{* *} p<0.001$ and ${ }^{* * *} p<0.0001$. 
between TMRM and TMRM with FCCP treated HEK293/ Kv1.3 cells compared with control HEK293 cells, indicating a more hyperpolarised MMP (Supplementary Fig. S10C). To investigate the role of Kv1.3 channels in the MMP phenotype, the mitochondrially-targeted Kv1.3 inhibitor PAPTP was added during TMRM incubation in both HEK293/Kv1.3 and control HEK293 cells. PAPTP did not affect the MMP, suggesting that the increased MMP observed in HEK293/Kv1.3 cells is not directly linked to mitochondrial Kv1.3 or the proliferative phenotype (Supplementary Fig. S10D).

Increased mitochondrial $\mathrm{Ca}^{2+}$ concentrations can drive dehydrogenase enzyme activity enhancing NADH availability (the principal OXPHOS electron donor) and increasing respiration. Both $\mathrm{Ca}^{2+}$ and NADH were measured in control HEK293 and HEK293/Kv1.3 cells as an indicator of mitochondrial redox status ${ }^{41}$. Cells were stained with the fluorescent $\mathrm{Ca}^{2+}$ indicator Rhod2-AM and analysed by flow cytometry. Cellular and mitochondrial $\mathrm{Ca}^{2+}$ levels were examined under two conditions; baseline Rhod2 fluorescence and Rhod2 fluorescence following addition of FCCP $(20 \mu \mathrm{M})$. The mitochondrial $\mathrm{Ca}^{2+}$ range can be estimated by examining the difference in Rhod2 fluorescence between baseline and FCCP treatment. Kv1.3 expression did not affect cellular $\mathrm{Ca}^{2+}$ concentrations (Supplementary Fig. S10E) or mean mitochondrial $\mathrm{Ca}^{2+}$ range in HEK293 cells (Supplementary Fig. S10F).

$\mathrm{NADH}$ concentrations measured by autofluorescence detection using flow cytometry were greater in HEK293/ Kv1.3 compared with control HEK293 cells (Supplementary Fig S10G). NADH autofluorescence was measured following $20 \mu \mathrm{M}$ FCCP treatment to maximally oxidise the $\mathrm{NAD}^{+} / \mathrm{NADH}$ pool, and both $0.5 \mu \mathrm{M}$ rotenone plus $2.5 \mu \mathrm{M}$ Antimycin A treatment to maximally reduce the $\mathrm{NAD}^{+} / \mathrm{NADH}$ pool (Supplementary Fig. S10G). NADH concentrations were higher in HEK293/Kv1.3 cells compared with control HEK293 cells in all conditions. The difference in fluorescence between the maximally oxidised and maximally reduced $\mathrm{NAD}^{+} / \mathrm{NADH}$ pools $(\mathrm{NADH}$ range) estimates total mitochondrial NADH and NAD ${ }^{+42}$. HEK293/Kv1.3 cells had an increased NADH autofluorescence range compared to control HEK293 cells (Supplementary Fig. S10H). HEK293/Kv1.3 cells were treated with $100 \mathrm{nM}$ PAPTP to inhibit Kv1.3 channels. PAPTP had no effect on NADH autofluorescence in HEK293/Kv1.3 cells (Supplementary Fig. S10I), suggesting that Kv1.3-induced increases in NADH do not mediate channel-enhanced respiration.

\section{Kv1.3 channels regulate proliferation and respiration via a non-conducting mechanism}

We interrogated the structural and functional properties of Kv1.3 that may regulate respiration. HEK293 cells were transfected to express mutant Kv1.3. Kv1.3-P89 is a voltage sensitive but non-conducting Kv1.3 channel with intact gating properties. It has a single point mutation (W389F) in the channel pore region (S5-S6) ${ }^{13}$. Kv1.3-P93 is a voltage insensitive and non-conducting Kv1.3 channel which shares the Kv1.3-P89 (W389F) point mutation and has three additional point mutations in the voltage sensor $(\mathrm{S} 4)^{13}$. This triple mutation (R320N, L321A and R326I) shifts the channel activation to potentials below $-170 \mathrm{mV}$. The channel is inactive at physiological voltages and functions as an inward rectifier ${ }^{43}$. Kv1.3 phosphorylation by intracellular kinases is a common posttranslational modification ${ }^{44,45}$. Kv1.3-P121 is voltage sensitive and conductance competent. However Kv1.3P121 is phosphorylation-defective due to a Y447A substitution in its C-terminus which prevents phosphorylation of this site by the extracellular signal-regulated kinases 1/2 (ERK1/2) $)^{13}$.

Using electrophysiology we confirmed that cells transfected with mutant Kv1.3 channels HEK293/Kv1.3-P89 and HEK293/Kv1.3-P93 are conductance deficient (Fig. 6A). HEK293/Kv1.3-P121 cells were conductance competent and exhibited comparable currents to HEK293/Kv1.3 cells (Fig. 6A).

We then determined the mechanism through which Kv1.3 channels regulate proliferation (Fig. 6B). Cells expressing Kv1.3 with a non-functioning pore (Kv1.3-P89) displayed increased proliferation when compared to control HEK293 cells. This suggests that ion conductance through the Kv1.3 channel is not essential for Kv1.3induced proliferation. Disruption of the Kv1.3 voltage sensor (Kv1.3-P93) ablated the channels effect on proliferation. HEK293/Kv1.3-P121 cells also did not exhibit increased proliferation compared to control HEK293 cells. These data indicate that Kv1.3-induced proliferation is regulated via a non-conducting mechanism requiring both an intact voltage sensor and the C-terminal ERK1/2 phosphorylation site.

We assessed whether Kv1.3 channels also regulate respiration via the non-conducting mechanism. Cells transfected with Kv1.3-P89, Kv1.3-P93 and Kv1.3-P121 were analysed with respirometry and compared to control HEK293 and HEK293/Kv1.3 cells. Expression of Kv1.3 increased cellular respiration (Fig. 6C). The Routine, Leak, ETS and ROX respiration of HEK293/Kv1.3-P89 cells was not significantly different from that of HEK293/Kv1.3 cells (Fig. 6C), suggesting that Kv1.3-mediated $\mathrm{K}^{+}$conductance is not necessary for Kv1.3-induced respiration. HEK293/Kv1.3-P93 cell Routine, Leak, ETS and ROX respiration was no different to control and reduced when compared to HEK293/Kv1.3 cells (Fig. 6D). These data are consistent with the observed reduction in proliferation in HEK293/Kv1.3-P93 cells compared with HEK293/Kv1.3 cells and suggest that the Kv1.3 voltage sensor is required for channel-induced respiration. Consistent with a 


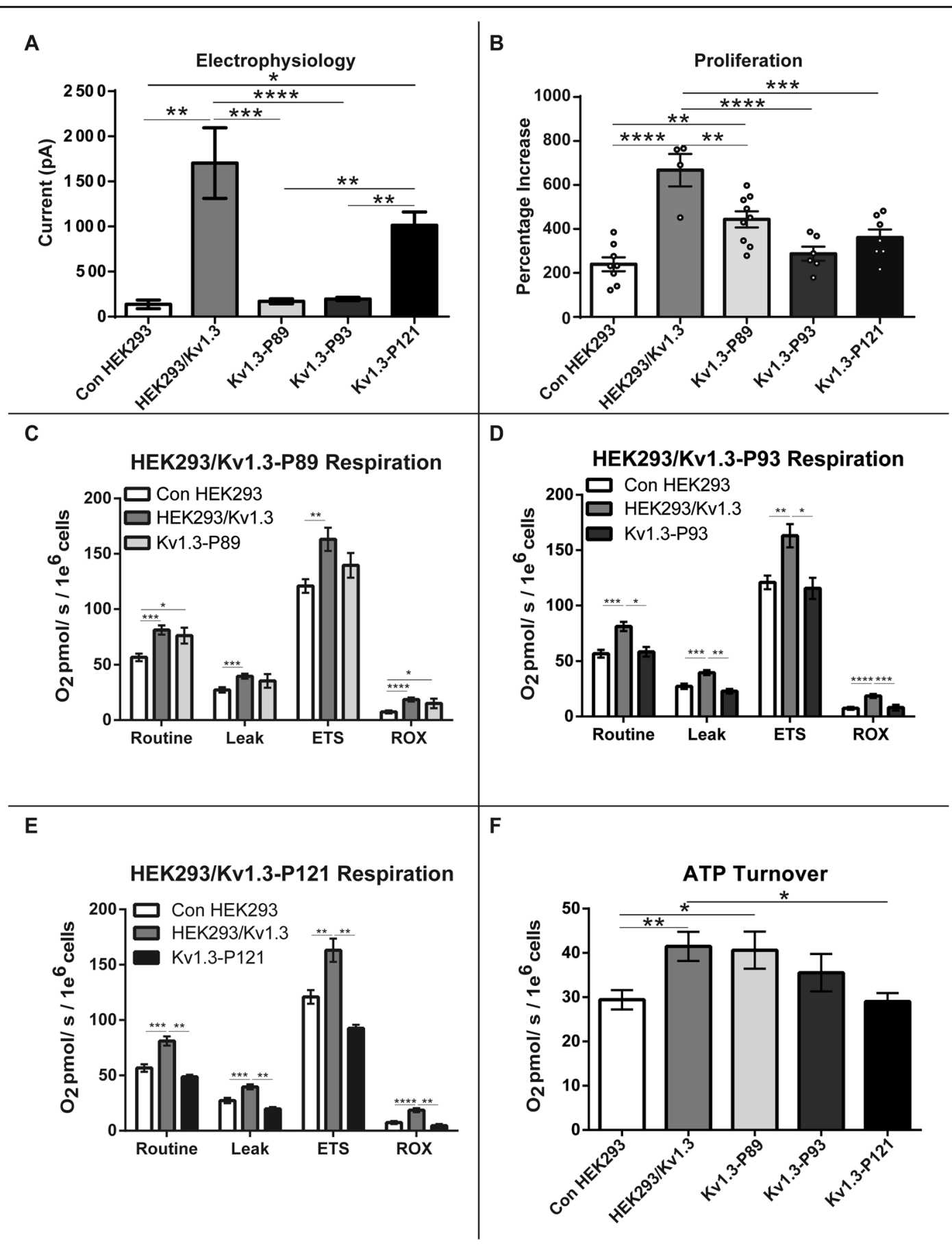

Fig. 6 Mitochondrial Kv1.3 channels regulate proliferation and respiration via a non-conducting mechanism. A Electrophysiology currents for control HEK293, HEK293/Kv1.3, HEK/Kv1.3-P89, HEK/Kv1.3-P93 and HEK293/Kv1.3-P121 when depolarised from $-80 \mathrm{mV}$ to $+40 \mathrm{mV}$ (control HEK293 cells, $n=6$; HEK293/Kv1.3 cells, $n=14$; HEK293/Kv1.3-P89 cells, $n=9$; HEK293/Kv1.3-P93 cells, $n=15 ;$ HEK293/Kv1.3-P121 cells, $n=23$ ). B Proliferation of control HEK293, HEK293/Kv1.3, HEK293/Kv1.3-P89, HEK293/Kv1.3-P93 and HEK293/Kv1.3-P121 cells expressed as a percentage increase in cell number over 3 days (control HEK293 cells, $n=8$; HEK293/Kv1.3 cells, $n=4$; HEK293/Kv1.3-P89 cells, $n=9$; HEK293/Kv1.3-P93 cells, $n=$ 6; HEK293/Kv1.3-P121 cells, $n=7$ ). C Routine, Leak, maximal electron transfer system (ETS) and residual (ROX) $\mathrm{O}_{2}$ consumption in control HEK293, HEK293/Kv1.3 and HEK293/Kv1.3-P89 cells (control HEK293, $n=27 ;$ HEK293/Kv1.3, $n=38 ;$ HEK293/Kv1.3-P89, $n=9$ ). D Routine, Leak, maximal ETS and ROX O 2 consumption in control HEK293, HEK293/Kv1.3 and HEK293/Kv1.3-P93 cells (control HEK293, $n=27$; HEK293/Kv1.3, $n=38 ;$ HEK293/Kv1.3-P93, $n=9$ ). E Routine, Leak, maximal ETS and ROX $\mathrm{O}_{2}$ consumption in control HEK293, HEK293/Kv1.3 and HEK293/Kv1.3-P121 cells (control HEK293, $n=$ 27; HEK293/Kv1.3, $n=38$; HEK293/Kv1.3-P121, $n=6$ ). F ATP turnover in control HEK293, HEK293/Kv1.3, HEK293/Kv1.3-P89, HEK293/Kv1.3-P93 and HEK293/Kv1.3-P121 cells (control HEK293 $n=27$; HEK293/Kv1.3 $n=38 ;$ HEK293/Kv1.3-P89 $n=9$; HEK293/Kv1.3-P93 $n=9 ;$ HEK293/Kv1.3-P121 $n=6$ ). Data were mean \pm SEM. Data were analysed using one-way ANOVA with Tukeys post hoc test, ${ }^{*} p<0.05,{ }^{* *} p<0.01,{ }^{* * *} p<0.001$ and ${ }^{* * * *} p<0.0001$. 
reduced rate of proliferation, Routine, Leak, ETS and ROX respiration in HEK293/Kv1.3-P121 cells was also reduced compared to HEK293/Kv1.3 cells (Fig. 6E), suggesting that preservation of the Y447 ERK1/2 phosphorylation site is essential for Kv1.3-induced respiration.

Cellular proliferation has a high energy demand, requiring increased ATP turnover. ATP turnover rate was calculated for control, Kv1.3, Kv1.3-P121, Kv1.3-P89 and Kv1.3-P93 HEK293 cells (Fig. 6F). Disruption of the channel pore (HEK293/Kv1.3-P89) had no effect on Kv1.3-enhanced ATP turnover. However, there was no difference between the ATP turnover in control HEK293 cells, HEK293/Kv1.3-P93 and HEK293/Kv1.3-P121 cells. Loss of a functioning voltage sensor (Kv1.3-P93) and the ERK1/2 phosphorylation site (Kv1.3-P121) ablates Kv1.3mediated enhanced ATP turnover.

\section{Discussion}

The Kv1.3 channel enhances cellular proliferation and has been implicated in diseases of pathological proliferation including cancer and cardiovascular disease ${ }^{26,46}$. Our data suggests Kv1.3 located in the mitochondria may be important to this phenotype. Proliferation is a highly energy demanding process requiring increases in ATP turnover. Kv1.3 channels induced an increase in cellular respiration and ATP turnover, independent of effects on mitochondrial content. However, Kv1.3 expression was not ubiquitously distributed among mitochondria. Whether the ion channel is enriched in a specific mitochondrial subpopulation remains to be determined.

Kv1.3-induced respiration had a direct mechanistic link to the proliferative phenotype beyond ATP generation. Kv1.3-driven OXPHOS increased the production of ROS which were required for proliferation. Using mutant Kv1.3 channels, we identified that Kv1.3-induced proliferation and respiration was independent of the channel pore. Mutation of the channel's voltage sensor ablated Kv1.3's effects on respiration and proliferation. Therefore the non-conducting process required to drive respiration remained linked to the channels' ability to sense the extracellular potential. The activity of intracellular ion channels can be regulated by kinase-mediated signalling cascades $^{13,44,45}$. Several pro-proliferative growth factors, including platelet-derived growth factor, signal via ERK to induce their cellular effects ${ }^{35}$. Kv1.3 has been identified as an ERK1/2 substrate with a tyrosine 447 phosphorylation site in the C-terminal region ${ }^{13}$. ERK signalling is required for Kv1.3-mediated proliferation ${ }^{13}$. Mutation of the phosphorylation site ablated the Kv1.3-induced increase in respiration and proliferation. This study proposes a mechanism for Kv1.3-mediated cellular proliferation whereby Kv1.3 channels require phosphorylation by ERK $1 / 2$ and the capacity to sense the extracellular potential to increase respiration, driving ROS production

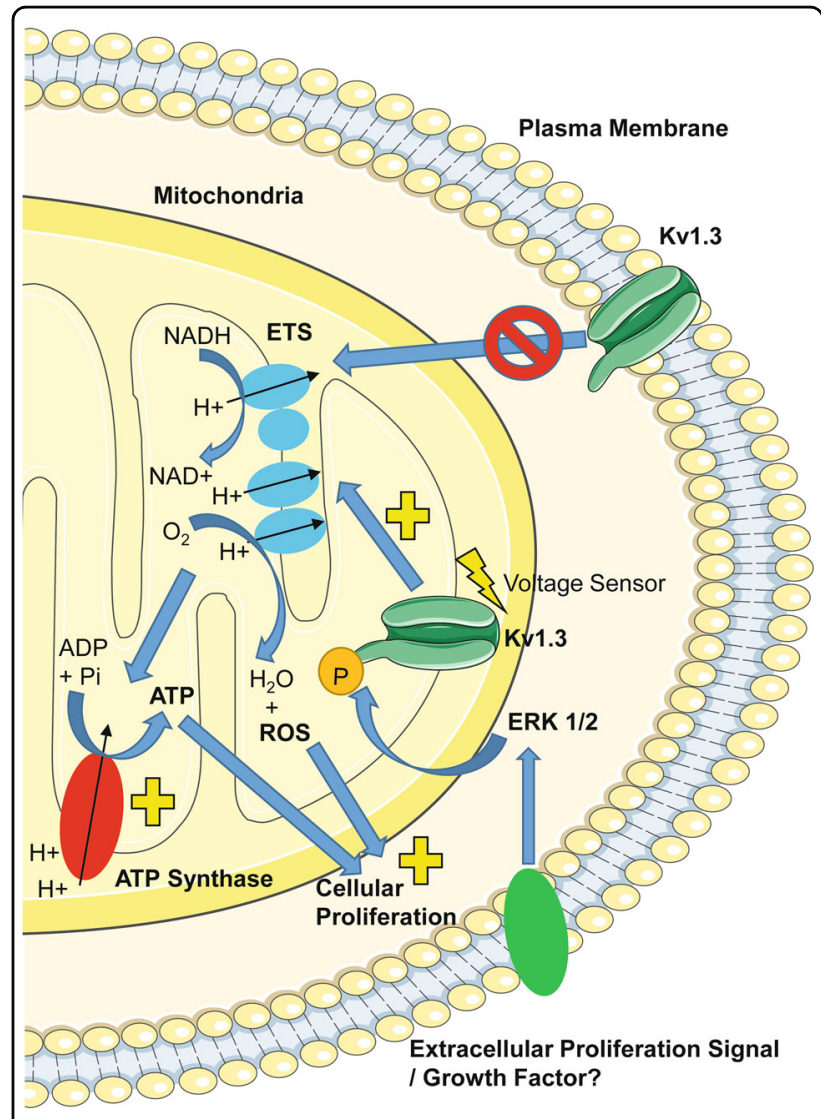

Fig. 7 Diagrammatic representation of the proposed mechanism for Kv1.3-induced respiration and proliferation. Kv1.3 channels potentially located at the mitochondria require voltage sensing and an intact ERK1/2 phosphorylation site (Y447) to stimulate

mitochondrial oxidative phosphorylation. Kv1.3-induced oxidative phosphorylation drives increased ATP turnover, meeting the increased energy demands needed for proliferation. Simultaneously, Kv1.3induced oxidative phosphorylation generates mitochondrial reactive oxygen species (ROS) which stimulate the proliferative phenotype of the cells. This process is independent of plasma membrane Kv1.3 channel ion conductance. However, it may be speculated that growth factor receptors at the cell plasma membrane may signal to Kv1.3 channels via downstream ERK1/2-mediated phosphorylation.

and, subsequently, proliferation (Fig. 7). The reliance on both voltage sensing and ERK phosphorylation for the regulation of respiration is consistent with a model in which, upon voltage sensing, Kv1.3 undergoes a conformational change exposing the ERK phosphorylation site in the C-terminus ${ }^{3,13,47}$. This is also consistent with the observation that PAPTP, which locks the channel in the inactive state, inhibits Kv1.3-induced respiration and proliferation. Kv1.3-induced respiration is not dependent on $\mathrm{K}^{+}$ion conductance; therefore PAPTPs inhibitory effects may instead relate to preventing the conformational change in Kv1.3 that exposes the ERK phosphorylation site at residue 447 . 
We acknowledge limitations to our study. Our data using the mitochondrially-targeted Kv1.3 inhibitor PAPTP suggest that mitochondrial Kv1.3 channels may be important to the Kv1.3-induced proliferative and respiration phenotype. However, future studies confirming this association and linking the Kv1.3 organelle location to the non-conducting mechanism, potentially through mitochondrially-targeted versions of the channel pore, voltage sensor and ERK phosphorylation-defective Kv1.3 channels will be important.

We identify that Kv1.3 coordinates cellular respiration to meet the energy requirements needed for proliferation. We suggest a mechanism linking ERK1/2 signalling to Kv1.3-mediated increased respiration, ROS generation and proliferation. Our study has inferences for growth factor-mediated mechanisms that converge on ERK to drive physiological and pathological proliferation.

\section{Acknowledgements}

We thank Professors José Ramón López López, and Teresa Pérez-García, University of Valladolid, for the gifts of the HEK293/Kv1.3 cell line and the plasmids containing the various WT and mutant Kv1.3 channels.

\section{Author details}

${ }^{1}$ School of Medicine, University of Leeds, Leeds LS2 9JT, UK. ${ }^{2}$ Faculty of Biological Sciences, University of Leeds, Leeds LS2 9JT, UK. ${ }^{3}$ School of Chemistry, University of Leeds, Leeds LS2 9JT, UK

\begin{abstract}
Author contributions
F.L.S. and M.M.A.-O. carried out the majority of experiments. J.L.S assisted with experiments throughout. E.C.-B. analysed confocal images. N.T.H. performed Western Blotting. R.S.B. and A.M. synthesised PAPTP. J.D.L. amplified the CDNA encoding the wild-type Kv1.5 channel. K.P. gave intellectual input to cell culture experiments. P.S. provided study supervision. C.P. designed and supervised the study. L.D.R. supervised the study and wrote the manuscript with input from all co-authors.
\end{abstract}

\section{Funding}

L.D.R. acknowledges support of the Diabetes UK RD Lawrence Fellowship (16/ 0005382), the Biotechnology and Biological Research Council (BB/R013500/1) and the Medical Research Council (MR/R014086/1). This work was funded by a British Heart Foundation PhD studentship (FS/14/41/30955). E.C.-B. was supported by a grant from the Wellcome Trust (Wellcome Trust grant 110044/ Z/15/Z). A.M. was supported by a BBSRC/AstraZeneca iCASE PhD studentship (BB/L015676/1). Confocal microscopy was supported by a Wellcome Trust Investigator Award received by Professor David Beech.

\section{Data availability}

All data and materials are available from the corresponding author on reasonable request.

\section{Ethics Statement}

This study did not require ethical approval.

\section{Conflict of interest}

The authors declare no competing interests.

\section{Publisher's note}

Springer Nature remains neutral with regard to jurisdictional claims in published maps and institutional affiliations.

Supplementary information The online version contains supplementary material available at https://doi.org/10.1038/s41419-021-03627-6.
Received: 24 November 2020 Revised: 9 March 2021 Accepted: 10 March 2021

Published online: 07 April 2021

\section{References}

1. Zhu, J. \& Thompson, C. B. Metabolic regulation of cell growth and proliferation. Nat. Rev. Mol. Cell Biol. 20, 436-450 (2019).

2. Efeyan, A., Comb, W. C. \& Sabatini, D. M. Nutrient-sensing mechanisms and pathways. Nature $\mathbf{5 1 7}, \mathbf{3 0 2 - 3 1 0}$ (2015).

3. Perez-Garcia, M. T., Cidad, P. \& Lopez-Lopez, J. R. The secret life of ion channels: Kv1.3 potassium channels and proliferation. Am. J. Physiol. Cell Physiol. 314, C27-C42 (2018)

4. Lang, F. et al. Cell volume regulatory ion channels in cell proliferation and cell death. Methods Enzymol. 428, 209-225 (2007).

5. Schwab, A., Fabian, A., Hanley, P. J. \& Stock, C. Role of ion channels and transporters in cell migration. Physiol. Rev. 92, 1865-1913 (2012).

6. $\mathrm{Xu}$, J. et al. The voltage-gated potassium channel Kv1.3 regulates peripheral insulin sensitivity. Proc. Natl Acad. Sci. USA 101, 3112-3117 (2004).

7. $\mathrm{Xu}$, J. et al. The voltage-gated potassium channel Kv1.3 regulates energy homeostasis and body weight. Hum. Mol. Genet. 12, 551-559 (2003).

8. Swanson, R. et al. Cloning and expression of cDNA and genomic clones encoding three delayed rectifier potassium channels in rat brain. Neuron 4, 929-939 (1990).

9. Bielanska, J. et al. Voltage-dependent potassium channels Kv1.3 and Kv1.5 in human cancer. Curr. Cancer Drug Targets 9, 904-914 (2009).

10. Grunnet, M. et al. KCNE4 is an inhibitory subunit to Kv1.1 and Kv1.3 potassium channels. Biophys. J. 85, 1525-1537 (2003).

11. Villalonga, N. et al. Cell cycle-dependent expression of Kv1.5 is involved in myoblast proliferation. Biochim. Biophys. Acta 1783, 728-736 (2008).

12. Bielanska, J. et al. Increased voltage-dependent $\mathrm{K}(+)$ channel Kv1.3 and Kv1.5 expression correlates with leiomyosarcoma aggressiveness. Oncol. Lett. 4, 227-230 (2012)

13. Jimenez-Perez, L. et al. Molecular determinants of Kv1.3 potassium channelsinduced proliferation. J. Biol. Chem. 291, 3569-3580 (2016).

14. Cidad, P. et al. Characterization of ion channels involved in the proliferative response of femoral artery smooth muscle cells. Arterioscler. Thromb. Vasc. Biol. 30, 1203-1211 (2010)

15. Cheong, A. et al. Potent suppression of vascular smooth muscle cell migration and human neointimal hyperplasia by KV1.3 channel blockers. Cardiovasc. Res. 89, 282-289 (2011).

16. Blackiston, D. J., McLaughlin, K. A. \& Levin, M. Bioelectric controls of cell proliferation: ion channels, membrane voltage and the cell cycle. Cell Cycle $\mathbf{8}$, 3527-3536 (2009).

17. Herrmann, S., Ninkovic, M., Kohl, T., Lorinczi, E. \& Pardo, L. A. Cortactin controls surface expression of the voltage-gated potassium channel KM10.1. J. Biol. Chem. 287, 44151-44163 (2012).

18. Cidad, P. et al. Kv1.3 channels can modulate cell proliferation during phenotypic switch by an ion-flux independent mechanism. Arterioscler. Thromb. Vasc. Biol. 32, 1299-1307 (2012).

19. Lee, A., Fakler, B., Kaczmarek, L. K. \& Isom, L. L. More than a pore: ion channel signaling complexes. J. Neurosci. 34, 15159-15169 (2014).

20. Oronsky, B. T. et al. Follow the ATP: tumor energy production: a perspective. Anticancer Agents Med. Chem. 14, 1187-1198 (2014).

21. Hoshi, T. \& Armstrong, C. M. C-type inactivation of voltage-gated K+ channels: pore constriction or dilation? J. Gen. Physiol. 141, 151-160 (2013).

22. Rasmusson, R. L. et al. Inactivation of voltage-gated cardiac K+ channels. Circ. Res. 82, 739-750 (1998).

23. Szabo, I. et al. A novel potassium channel in lymphocyte mitochondria. J. Biol. Chem. 280, 12790-12798 (2005)

24. Jang, S. H. et al. Nuclear localization and functional characteristics of voltagegated potassium channel Kv1.3. J. Biol. Chem. 290, 12547-12557 (2015).

25. Gulbins, E., Sassi, N., Grassme, H., Zoratti, M. \& Szabo, I. Role of Kv1.3 mitochondrial potassium channel in apoptotic signalling in lymphocytes. Biochim. Biophys. Acta 1797, 1251-1259 (2010).

26. Leanza, L. et al. Direct pharmacological targeting of a mitochondrial ion channel selectively kills tumor cells in vivo. Cancer Cell 31, 516-531 e510 (2017).

27. Al-Owais, M. M. et al. Multiple mechanisms mediating carbon monoxide inhibition of the voltage-gated $\mathrm{K}(+)$ channel Kv1.5. Cell Death Dis. 8, e3163 (2017). 
28. Duckles, $\mathrm{H}$. et al. Heme oxygenase-1 regulates cell proliferation via carbon monoxide-mediated inhibition of T-type Ca2+ channels. Pflug. Arch. 467, 415-427 (2015).

29. Elies, J., Johnson, E., Boyle, J. P., Scragg, J. L. \& Peers, C. H2S does not regulate proliferation via T-type Ca2+ channels. Biochem. Biophys. Res. Commun. 461, 659-664 (2015)

30. Duckles, $\mathrm{H}$. et al. T-type Ca2+ channel regulation by CO: a mechanism for control of cell proliferation. Adv. Exp. Med. Biol. 860, 291-300 (2015).

31. Garnham, J. O. et al. Divergent skeletal muscle mitochondrial phenotype between male and female patients with chronic heart failure. J. Cachexia Sarcopenia Muscle 11, 79-88 (2019).

32. Hall, A. \& Moghimi, S. M. Determination of polycation-mediated perturbation of mitochondrial respiration in intact cells by high-resolution respirometry (Oxygraph-2k, OROBOROS). Methods Mol. Biol. 1943, 313-322 (2019).

33. Larsen, S. et al. Biomarkers of mitochondrial content in skeletal muscle of healthy young human subjects. J. Physiol. 590, 3349-3360 (2012).

34. Roberts, L. D. et al. Inorganic nitrate promotes the browning of white adipose tissue through the nitrate-nitrite-nitric oxide pathway. Diabetes $64,471-484$ (2014).

35. Cidad, P. et al. Kv1.3 channels modulate human vascular smooth muscle cells proliferation independently of mTOR signaling pathway. Pflug. Arch. 467, 1711-1722 (2015).

36. Rashid, M. H. et al. A potent and selective peptide blocker of the Kv1.3 channel: prediction from free-energy simulations and experimental confirmation. PLOS ONE 8, e78712 (2013).
37. Leanza, L., Zoratti, M., Gulbins, E. \& Szabo, I. Induction of apoptosis in macrophages via Kv1.3 and Kv1.5 potassium channels. Curr. Med. Chem. 19, 5394-5404 (2012).

38. Mattarei, A. et al. Novel mitochondria-targeted furocoumarin derivatives as possible anti-cancer agents. Front. Oncol. 8, 122 (2018).

39. Sauer, H., Wartenberg, M. \& Hescheler, J. Reactive oxygen species as intracellular messengers during cell growth and differentiation. Cell Physiol. Biochem. 11, 173-186 (2001).

40. O'Rourke, B. Mitochondrial ion channels. Annu. Rev. Physiol. 69, 19-49 (2007).

41. Bonuccelli, G., De Francesco, E. M., de Boer, R., Tanowitz, H. B. \& Lisanti, M. P. $\mathrm{NADH}$ autofluorescence, a new metabolic biomarker for cancer stem cells: identification of Vitamin C and CAPE as natural products targeting "stemness". Oncotarget 8, 20667-20678 (2017).

42. Buckler, K. J. \& Turner, P. J. Oxygen sensitivity of mitochondrial function in rat arterial chemoreceptor cells. J. Physiol. 591, 3549-3563 (2013).

43. Miller, A. G. \& Aldrich, R. W. Conversion of a delayed rectifier K+ channel to a voltage-gated inward rectifier $\mathrm{K}+$ channel by three amino acid substitutions. Neuron 16, 853-858 (1996).

44. Chung, I. \& Schlichter, L. C. Native Kv1.3 channels are upregulated by protein kinase C. J. Membr. Biol. 156, 73-85 (1997).

45. Chung, I. \& Schlichter, L. C. Regulation of native Kv1.3 channels by CAMPdependent protein phosphorylation. Am. J. Physiol. 273, C622-C633 (1997).

46. Jackson, W. F. KV1.3: a new therapeutic target to control vascular smooth muscle cell proliferation. Arterioscler. Thromb. Vasc. Biol. 30, 1073-1074 (2010).

47. Lopez-Lopez, J. R., Cidad, P. \& Perez-Garcia, M. T. Kv channels and vascular smooth muscle cell proliferation. Microcirculation 25, e12427 (2018). 